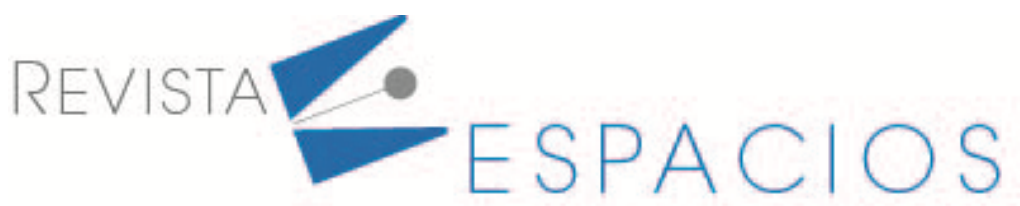

\title{
La necesidad de establecer políticas públicas gubernamentales en Ecuador para garantizar el derecho a la salud de los adultos mayores
}

\section{Government public policies for access to the right to health of older adults}

\author{
MONJE, Enma A. ${ }^{1}$
}

SAN LUCAS, Maria F. ${ }^{2}$

\section{Resumen}

El derecho a la salud está garantizado por el Estado ecuatoriano, sin embargo, las instituciones que se encuentran a cargo de responder por este, no garantizan la accesibilidad oportuna para los adultos mayores. Por ello, es importante analizar las políticas públicas dirigidas a este sector. La metodología a utilizar en la investigación es descriptiva. A través de métodos teóricos, se busca alcanzar resultados que determinen la atención prioritaria por parte de las instituciones estatales que ofrecen el servicio de salud.

Palabras clave: políticas publicas, adultos mayores, salud

\begin{abstract}
The right to health is guaranteed by the Ecuadorian State, however, the institutions in charge of responding for it do not guarantee timely accessibility for the elderly. For this reason, it is important to analyze the public policies directed to this sector, the methodology to be used in the research is descriptive through theoretical methods, it seeks to achieve results that determine the priority attention on the part of the state institutions that offer the health service.
\end{abstract}

Key words: public policies, seniors, health

\section{Introducción}

Las políticas públicas son consideradas como herramientas para resolver problemáticas de determinados grupos de personas (Aguilar 2015); en tal sentido, una política pública es un medio usado por los Estados usan para garantizar el efectivo cumplimiento de los derechos de las personas. Para la Organización Panamericana de la Salud (1997), las políticas públicas, deben priorizar a los sectores más vulnerables, sin excluir a ningún grupo, sobre todo de aquellos considerados como vulnerables.

\footnotetext{
${ }^{1}$ Estudiante de la Maestria en Derecho,mención Gestión Pública. Pontificia Universidad Católica. Ecuador sede Ambato. Correo electrónico de contacto:aliciamonje2012@hotmail.com.

${ }^{2}$ Docente.Pontificia Universidad Católica. Ecuador sede Ambato. Correo electrónico de contacto: msanlucas@pucesa.edu.ec
} 
De acuerdo con Abarca (2002), a nivel mundial, durante los años noventa, algunos países implementaron políticas en beneficio de los adultos mayores y procedieron a la promulgación de leyes específicas para este grupo de personas, y realizar reformas a nivel constitucional en las cuales se incorporaron beneficios para los adultos mayores. Fruto de estos procesos, se da el reconocimiento del año internacional de las personas mayores en la Asamblea Mundial sobre el Envejecimiento, realizada en Madrid en el año 2002. Se prevé que la aplicación de la Estrategia Regional impulsará el desarrollo de nuevas iniciativas en favor de las personas mayores en el futuro cercano.

De lo que establece en la Constitución de la República del Ecuador (2008), en el artículo 141, corresponde a los ministerios del Estado, ejercer la rectoría de las políticas públicas del área a su cargo basadas en un enfoque de Derechos Humanos.

Las políticas públicas orientadas a los adultos mayores y su accesibilidad al sistema de salud pública, en Ecuador, suelen ser deficientes, debido a los engorrosos trámites administrativos. Conforme lo manda la Constitución de la República del Ecuador, el derecho a la salud específicamente de los adultos mayores, debería ser gratuito, seguro y eficiente, pero esto no se cumple, por lo que es necesario establecer políticas públicas eficaces que permitan alcanzar su cumplimiento.

Para Duval (2019), la salud se refiere a aspectos como: vida digna, calidad alimentaria, accesos a servicios básicos, vivienda y condiciones óptimas para desempeño laboral, además es un derecho reconocido tanto en la Constitución como en los Tratados Internacionales. Por ello el Estado debe disponer de políticas pública orientadas a mejorar este problema crítico que se afronta en la actualidad.

Esto amerita ser investigado y reviste importancia puesto que implica la accesibilidad a un servicio integral que afecta los aspectos de la vida cotidiana de los adultos mayores y por tanto, se puede alertar y prohibir conductas que se perciban como riesgosas, alcanzar mayores beneficios, precautelar derechos y realizar actividades que beneficien a este importante grupo de la sociedad. Por estas razones consideramos necesario realizar un estudio de las políticas existentes en Ecuador, para plantear soluciones a las necesidades de los diferentes sectores que requieren mayor atención por parte del sistema sanitario, en particular para las personas adultas mayores.

\subsection{Políticas Públicas}

Huenchuan (2009), refiere a las Políticas Públicas como un factor importante en el manejo gubernamental, sobre el acceso que brinde a servicios y bienes de calidad especialmente sobre determinados grupos y personas que forman parte de una sociedad. Por otro parte, Cicogna (2020), menciona que las políticas públicas, son una serie de normas y respuestas que permiten al Estado tener injerencia en determinados sectores significativos para la sociedad para brindar soluciones agiles y oportunas a ciertas problemáticas de determinados grupos de personas.

En este sentido, Montenegro (2020), establece que las políticas públicas son soluciones específicas planteadas por autoridades del Estado para frenar problemáticas que afectan gravemente los derechos de las personas, en especial de los grupos más vulnerables. Finalmente, con relación específica al tema a tratar es importante mencionar lo dicho por Huenchuam (1999), citado por Guzmán (2005, p.14), "las políticas de vejez son aquellas acciones organizadas por parte del Estado frente a las consecuencias sociales, económicas y culturales del envejecimiento poblacional e individual". 


\subsection{Políticas Públicas de atención al adulto mayor}

En el año 2007 el Ministerio de Salud Pública (MSP) promulgó la política de salud, centrada en la familia y la comunidad, con enfoque intercultural de género, intergeneracional y territorial, basado en los principios de la atención primaria en salud, acorde a los derechos y deberes establecidos en la Constitución de la República del Ecuador. En el antes mencionado modelo se toma en cuenta a los adultos mayores como sujetos de derechos y de atención prioritaria.

La Política Nacional de Salud en Ecuador, en relación al grupo de adultos y adultos mayores, busca descentralizar la responsabilidad del Ministerio de Salud Pública, como ente rector de la salud, dando estas competencias a las siguientes instituciones: la Dirección Nacional de Gerontología del Ministerio de Inclusión Social, de cada provincia, el Instituto Ecuatoriano de Seguridad Social, la Dirección Nacional del Adulto Mayor, para brindar atención a los jubilados. En cada provincia del país existe un delegado que, en esta dirección, elabora los programas y proyectos de atención a los jubilados. Por otra parte, los Gobiernos Autónomos Descentralizados tienen como obligación incluir programas y proyectos para atender a los adultos mayores no jubilados y se encuentra regulado por el artículo 19 de la Ley del Adulto Mayor.

\subsection{Adulto mayor}

Zafra (2006), menciona que la Organización Mundial de la Salud considera a las personas de 60 a 74 años como edad avanzada y de 75 a 90 años ancianos, y los mayores de 90 se les denomina grandes longevos. Además refiere que las personas mayores de 60 años se le llamará personas de la tercera edad; sin embargo, esto dependerá mucho del país y la calidad de vida de la persona. Esta es una realidad en la cual se consideran aspectos sociales, culturales y fisiológicos. En el siglo XIII se consideraba que una persona era vieja a los de treinta años, en el siglo XIX, se consideraba vieja a los cuarenta años, sin embargo, en la actualidad la edad a partir de la cual una persona es considerada como un adulto mayor está en entredicho.

Los adultos mayores en Ecuador representan un $4.2 \%$ de la población total y se estima que para el 2050, alcance al 16\%. La primera encuesta de Salud, Bienes y Envejecimiento (SABE I), permite realizar un análisis de las condiciones de salud en las que se encuentran los adultos mayores, Alli se verificó que un tercio vive en condiciones económicas malas, puesto que si bien percibe recursos de sus familiares deben buscar por sus propios medios la manera de sobrevivir, lo que implica que tengan limitado servicio de salud (Carrión, 1986; INEC, 2011).

Se debe agregar que la realidad del adulto mayor en Ecuador es lamentable, puesto que de acuerdo al Censo de Población y Vivienda del INEC 2010, se establece que el 57,4 \% de la población adulta mayor se encuentran en una condición de extrema pobreza. Esto demuestra que los adultos mayores de Ecuador viven en una situación socio económico deplorable y no pueden aspirar a una vejez digna. Un alto porcentaje de esta población no cuenta con ingresos económicos fijos como el de una jubilación o pensión y se ven en la obligación de trabajar en su vejez.

\subsection{Derechos de los adultos mayores}

En la Convención Interamericana de Derechos Humanos (1977), se menciona la protección de los derechos humanos de los adultos mayores y el derecho a la vida, a la independencia, la seguridad, educación, salud, entre otros, que deberían ser respetados y cumplidos de manera obligatoria por los Estados.

En la Declaración Universal de Derechos Humanos (1948), figuran algunos derechos fundamentales como la igualdad y la prohibición de discriminación por cualquier condición, el derecho a la seguridad social, a un nivel 
de vida adecuada, asistencia médica y a los servicios sociales necesarios. Todos estos derechos son extensibles a las personas adultas mayores y de importancia fundamental para el desarrollo de las libertades en la vejez.

El Protocolo de San Salvador (1988), en el artículo 17, establece de manera específica medidas a favor de los adultos mayores, también las Asambleas Mundiales sobre el Envejecimiento (Viena 1982 y Madrid 2002), exhortan a los gobiernos que incorporen los veinte principios de las Naciones Unidas a favor de las personas de edad que se refieren a tener acceso alimentación, vivienda, vestimenta, salud, oportunidad de trabajo, acceso a programas de educación, integración en la sociedad, aplicación de políticas favorables, promover grupos asociaciones, acceso a servicios sociales que le permitan mantener un nivel físico y metal adecuado. El Protocolo de San Salvador es un instrumento vinculante para los países de América Latina y el Caribe, por lo que sus disposiciones son de obligatorio cumplimiento para los Estados.

El Pacto de Derechos Económicos, Sociales y Culturales (1966), en el artículo 3, menciona la obligación de los Estados de brindar una atención especial a los adultos mayores, creando subsidios o cualquier tipo de ayuda, especialmente para el acceso al servicio de salud y otros servicios básicos que son indispensables para alcanzar un nivel de vida óptimo.

La Constitución de la República del Ecuador (2008), en el artículo 23, numeral 2, determina que el Estado ecuatoriano debe adoptar medidas para erradicar y evitar cualquier tipo de violencia contra las personas de la tercera edad, en concordancia con el artículo 53 que dispone que los adultos mayores tienen derecho a asistencia especial que les asegure un nivel de vida digno, atención integral de salud gratuita y tratamiento preferente.

Es importante señalar que, en la ley del anciano (2006), refiere que: el Estado protegerá de modo especial, a los ancianos abandonados o desprotegidos, asimismo, fomentará y garantizará el funcionamiento de instituciones del sector privado que cumplan actividades de atención a la población adulta mayor, con sujeción a la presente Ley, en especial a aquellas entidades, sin fines de lucro, que se dediquen a la constitución, operación y equipamiento de centros hospitalarios gerontológico y otras actividades similares (art.3).

De acuerdo a la normativa antes mencionada corresponde a los Estados, adoptar los diferentes mecanismos para hacer efectivos los derechos de los adultos mayores, mismos que se encuentran reconocidos tanto en la Carta Magna como en las leyes especiales.

\subsection{El derecho al acceso a la salud de los adultos mayores}

La Organización Mundial de la Salud define la salud como un estado de completo bienestar físico, mental y social y no meramente como la ausencia de enfermedades o padecimientos. Llegar a la vejez gozando de buena salud y bienestar requiere un esfuerzo personal durante toda la vida y un entorno en el que ese esfuerzo pueda tener éxito.

Se debe agregar que en la Declaración Universal de los Derechos Humanos (1948), en su artículo 25, describe que todo ser humano tiene derecho a un nivel de vida adecuada que asegure la salud, la asistencia médica, los servicios sociales necesarios y el acceso a seguros, en consecuencia, el antes mencionado derecho es inherente al bienestar, sin la adecuada cobertura, no se puede acceder al servicio.

La Declaración Americana de los Derechos y Deberes del Hombre (1948), en su artículo 11, refiere que toda persona tiene derecho a preservar su salud, la asistencia médica y sanitaria de acuerdo a la disponibilidad de los recursos públicos; en este artículo se puede deducir que el principal responsable de suministrar el servicio sanitario es el Estado independientemente de las posibilidades económicas de cada individuo. 
El Pacto Internacional de Derechos Económicos, Sociales y Culturales (1966), establece que el disfrute de la salud debe darse en su nivel más alto posible, bajo dos aristas, la mental y la física, es decir, no basta la armonía del cuerpo como existencia orgánica, sino también de la psíquica, de modo que, los Estados se comprometen a la creación de condiciones que asegure a todos los ciudadanos la asistencia médica y servicios médicos en caso de enfermedad, sin importar su edad o su condición económica.

Se debe agregar que el Estado debe garantizar el derecho a la salud de los adultos mayores que tengan una atención prioritaria y especializada, ya sea, en las casas de salud públicas o privadas, poner énfasis en el ámbito de la inclusión social, económica, y protección contra la violencia; así también, son reconocidas como personas adultas mayores aquellas personas que han cumplido los sesenta y cinco años de edad.

Cabe mencionar, además, que este grupo considerado como vulnerable tienen los siguientes derechos: atención gratuita y especializada de salud, así como el acceso gratuito a medicinas; el trabajo remunerado en función de sus capacidades, para lo cual tomará en cuenta sus limitaciones; la jubilación universal; rebajas en los servicios públicos y en servicios privados de transporte y espectáculos; exenciones en el régimen tributario; exoneración del pago por costos notariales y registrales, de acuerdo con la ley; el acceso a una vivienda que asegure una vida digna, respeto a su opinión y consentimiento, según los artículos 32, 36, 37 de la Constitución de la República del Ecuador (2008).

La Ley Orgánica de las personas adultos Mayores (2019), en los artículos 9,46 y 59, menciona que uno de los deberes principales del Estado es brindar una atención prioritaria a los adultos mayores tanto en las instituciones públicas como privadas, sobre todo aquellos que padecen enfermedades catastróficas, además refiere que tienen derecho a recibir medicamentos de manera gratuita y el acceso al servicio de salud de manera oportuna y de calidad.

El Estado ecuatoriano tiene la obligación de garantizar la salud de toda su población; cabe señalar que, se ha determinado niveles de desigualdad, específicamente en los adultos mayores, sin embargo, en los últimos años se observa un descenso importante; mientras el índice de Gini a finales de 2003 fue 0.627, para finales de 2011, llego a 0.473 según la Secretaria Nacional de Planificacion y Desarrollo - Instituto Nciional de Estadisticas y Censos (SENPLADES-INEC, 2011).

Huenchuan (2009), menciona que el acceso al derecho a la salud y a la calidad de vida de las personas adultas mayores se ve limitada por dos factores, el nivel educación y el nivel socioeconómico. A mayor nivel de educación las personas tienen mayor posibilidad de acceder a un trabajo mejor remunerado y acceso al seguro universal; mientras que, las personas con menor nivel educativo, tienen menor posibilidad de acceder al servicio de salud, debido a que, obtienen trabajos informales y ningún tipo de seguro social.

De lo descrito en el párrafo anterior se puede colegir que, las personas adultas mayores tienen mayor posibilidad de obtener una mejor calidad de vida al llegar a su vejez, gracias a su propio esfuerzo y no a una política pública o programa del gobierno central. Al llegar a la vejez los únicos ingresos que tienen las personas del sector rural son el apoyo familiar y lo poco que pueden producir en sus tierras.Por ello, el acceso a la salud no es equitativa

Cuando preguntan a las Personas Adultas Mayores (PAM) sobre su percepción de su condición de salud se hace evidente que a medida que se incrementa la edad, la percepción de mala salud se incrementa y alcanza (entre las personas de 75 años y más de edad) a un cuarto del grupo de encuestados. En este contexto, las percepciones son importantes porque son hechos sociales que afectan en forma directa e indirecta las decisiones y acciones que se toman frente a las condiciones objetivas de la salud (SABE, 2009-2010). 


\section{Metodología}

\subsection{Tipo de Investigación}

El tipo de investigación utilizada fue la descriptiva y se centra en medir los conceptos o variables a los que se refieren, con la mayor precisión posible, con la finalidad de obtener las opiniones y puntos de vista de las personas, Hernández (2003).

\subsection{Enfoque de la investigación}

El enfoque utilizado fue el cualitativo. La presente investigación se basa en datos demográficos de estudios previos y de información descargada de los organismos oficiales internacionales como: la Comisión Económica para América Latina(CEPAL), Organización Iberoamericana de Seguridad Social (OISS), organismos oficiales nacionales, Instituto Ecuatoriano de Estadísticas y Censos (INEC), La encuesta nacional de salud, bienestar y nutrición de las personas adultas mayores ecuatorianas, que se realizó por primera vez en 2010, el Ministerio de Inclusión Económica y Social (MIES) y en libros relacionados con la temática para valorar la accesibilidad que tienen los adultos mayores a los servicios de salud.

\subsection{Nivel de investigación}

La investigación relaciona tres parámetros investigativos: descriptiva, transversal y social.

Es descriptiva, porque permite investigar una situación especifica, lo más importante en relación a la aplicación de las políticas públicas gubernamentales en relación a los adultos mayores. Transversal, por lo que a través de la investigación se busca analizar la problemática existente en Ecuador y, por otra parte, es macro social porque comprende a todo el país, puesto que es importante realizar un análisis de las Políticas Públicas Gubernamentales en relación a toda la poiblación de los adultos mayores.

\section{Resultados y discusión}

Estudios realizados demuestran que la población adulta mayor es cada vez más extensa. Esto se da a consecuencia los avances en prevención y atención en salud en algunos naciones, al descenso de la mortalidad y al aumento de la esperanza de vida que al transcurrir el tiempo se incrementa. Se debe agregar que, según estudios y análisis de tendencias del crecimiento de la población envejecida, esta representa el $10 \%$ de la población de Latinoamérica. Resultados parecidos se obtuvieron en Perú y Ecuador, según la Encuesta Demográfica y de Salud Materno Infantil, ENDEMAIN (2004). Las personas adultas mayores que no acceden al derecho al servicio de salud por falta de recursos económicos, llega al 68\% entre los 60 a 74 años y al $82 \%$ en las personas mayores de 75 años. Además, la encuesta indica que el 80,8\% de los ecuatorianos no tienen ningún tipo de seguro; solo el 19,2 \% están afiliados, de estos el 7,5\% están cubiertos por el seguro general del Instituto Ecuatoriano de Seguridad Social (IESS), y el 7,7\% por el Seguro Social Campesino (SSC).

El Ecuador es considerado como un país con un buen nivel de desarrollo; según el Programa de las Naciones Unidas para el Desarrollo PNUD (2011), su índice de desarrollo en 2011 fue de .720, que coloca el país en el puesto 83 de 187 naciones. Sin embargo, todavía persisten altos grados de desigualdad, que afectan de manera directa a una proporción considerable de las PAM. 
Cuadro 1

Indice de condiciones sociales de las personas adultas mayores por región

\begin{tabular}{|c|c|c|c|c|c|c|}
\hline \multirow{2}{*}{ Subregión } & \multicolumn{7}{|c|}{ Índice de condiciones socioeconómicas (\%) } \\
\cline { 2 - 7 } & $\begin{array}{c}\text { Muy } \\
\text { Buenas }\end{array}$ & Buenas & Regulares & Malas & Indigentes & Total \\
\hline Sierra urbana & 12.7 & 19.7 & 52.6 & 2.1 & 12.9 & 100.0 \\
\hline Sierra rural & 0.9 & 4.9 & 27.4 & 17.9 & 48.9 & 100.0 \\
\hline Costa urbana & 7.8 & 11.2 & 54.3 & 4.6 & 22.1 & 100.0 \\
\hline Costa rural & 1.1 & 4.2 & 34.0 & 22.3 & 38.3 & 100.0 \\
\hline Quito & 31.8 & 24.8 & 37.3 & 2.1 & 4.0 & 100.0 \\
\hline Guayaquil & 16.8 & 10.3 & 54.8 & 10.4 & 7.6 & 100.0 \\
\hline Total & 10.9 & 12.1 & 43.8 & 9.8 & 23.5 & 100.0 \\
\hline
\end{tabular}

Fuente: Freire (2010)

Se deduce en el cuadro 1 que un tercio de las PAM viven en condiciones malas o son indigentes; siendo la proporciones más alta en el área rural, especialmente en la sierra.

Al observar los datos de Quito y Guayaquil, se determina que una mayor proporción de las PAM se ubica en los estratos de condiciones muy buenas y buenas, a la vez que la proporción que vive en condiciones malas o de indigencia son más bajas. También se nota que Quito tiene una tasa más alta de PAM que vive en condiciones buenas o muy buenas que Guayaquil, así también se determina que el $68,3 \%$ de la población adulta mayor no tiene ningún seguro de salud, mientras solo el $28,2 \%$ son beneficiarios del sistema de seguridad social, porque formaron parte, en su vida laboral, del sector formal de trabajo.

Cuadro 2

Lugar al cual acuden los adultos mayores al considerse enfermos en el último año por grupo de edad (\%).

\begin{tabular}{|l|c|c|c|c|}
\hline \multicolumn{1}{|c|}{ Lugar de atención } & $\mathbf{6 0}$ a $\mathbf{6 4}$ & $\mathbf{6 5}$ a 74 & $\mathbf{2 7 5}$ & Total \\
\hline No estuvo enfermo & 18.0 & 16.5 & 12.9 & 15.7 \\
\hline Enfermo, pero no consultó & 6.2 & 7.0 & 6.7 & 6.7 \\
\hline Establecimiento público & 29.8 & 29.1 & 30.9 & 29.9 \\
\hline Establecimiento Privado & 37.7 & 39.3 & 39.3 & 38.9 \\
\hline Botica & 2.7 & 2.4 & 2.8 & 2.6 \\
\hline Naturista & 1.0 & 1.5 & 2.2 & 1.6 \\
\hline Otro & 4.6 & 4.1 & 5.3 & 4.7 \\
\hline \multicolumn{1}{|c|}{ Total } & 100.0 & 100.0 & 100.0 & 100.0 \\
\hline
\end{tabular}

Fuente: Freire (2010)

En el cuadro 2 se desprende primero que uno de cada cinco PAM reporta que no se enferma o que, al enfermarse, no acude a ningún servicio de salud. Esto se debe a que no tienen los recursos necesarios o se auto medican. Segundo, se observa que una proporción más alta de PAM en todos los grupos de edad prefiere acudir a un servicio privado que un servicio público que, al menos nominalmente, es gratuito. Esto se da por la mejor calidad, atención y accesibilidad al servicio sanitario privado.

El uso de servicios privados de salud, contextualizo con la información presentada en el cuadro, demuestra que la razón principal (de dos de cada tres PAM) por no acudir a un servicio ambulatorio de salud es la falta de dinero. Este hallazgo no solamente refleja el uso de servicios privados en donde el paciente paga todos los costos, sino también los servicios médicos públicos, cuyo uso implica costos directos. 
Cuadro 3

Razón por la que no acude al servicio médico

\begin{tabular}{|l|c|}
\hline \multicolumn{1}{|c|}{ Razón } & \% de respuestas \\
\hline Falta de transporte & 2.5 \\
\hline No tuvo tiempo & 2.0 \\
\hline No tiene dinero & 67.5 \\
\hline La atención no es buena & 4.7 \\
\hline Otras razones & 23.3 \\
\hline Total & $\mathbf{1 0 0 . 0}$ \\
\hline
\end{tabular}

Fuente: Freire (2010)

Del cuadro 3 se puede establecer que más del $50 \%$ de la población adulta mayor no puede acceder al servicio de salud por no contar con suficientes ingresos económicos. Con este dato se puede determinar lo importante que el Estado ecuatoriano establezca una política pública eficaz para dar solución a esta problemática.

Se puede establecer de acuerdo a los párrafos anteriores que el factor determinante para el acceso al derecho a la salud de los adultos mayores es el económico y que también depende cuan asequible son los sistemas de salud de cada país. Por ello, los gobiernos deben enfocarse en la implementación de políticas públicas y programas que ayuden a las personas adultas mayores y que puedan obtener ingresos adecuados de acuerdo a las necesidades propias de su edad.

Los sistemas de salud deben ser más accesibles a las personas de los grupos vulnerables, en especial para las personas adultas mayores, que dan lo mejor de sus años y aportan al desarrollo de su país; se puede mejorar la accesibilidad y la atención con la eliminación de la burocracia, la especialización de los profesionales en geriatría, incentivar a los jóvenes hacia el estudio de geriatría, capacitar de manera permanente a los profesionales que se encuentran al servicio de las personas adultas mayores y la eliminación de las desigualdades de acceso; por lo que los Estados, fundamentándose en las normas internacionales y nacionales de cada país, deben garantizar el derecho a la accesibilidad y la calidad del servicio de salud especializada y gratuita para las PAM.

En la situación delicada que se vive en la actualidad debido a la pandemia del COVID-19, se ha puesto a prueba el sistema de salud de los diferentes países del mundo y la accesibllidad que tienen a ellos especialmente los grupos vulnerables. Se puede deducir que el nivel socio económico es importante para acceder a una atención y medicamentos de calidad para solventar su situación de salud por la pandemia, mientras que las personas que tienen bajos ingresos económicos se auto medican o simplemente mueren en sus hogares. "Las Personas Mayores tienen mayor mortalidad ante esta infección. Hasta el 15 de abril del 2020 el $66 \%$ de fallecidos eran mayores de 60 años, siendo la letalidad en este grupo poblacional de $8,8 \%$ mientras que la letalidad general es de 2,\% (Parodi \& Runzer, 2020).

La población adulta mayor en Ecuador es el 10\% de la población en general, o sea alrededor de 1.662 .000 personas. De acuerdo el Boletín Estadístico del INEC de 2018, 468.717 están registrados como pensionistas de jubilación, en el Instituto Ecuatoriano de Seguridad Social (IESS) sea por montepío, invalidez, riesgos de trabajo y por vejez y el número de jubilados es 359.113. Estas cifras demuestran que, del total de la población adulta mayor, solo el $21.60 \%$, gozan de un sustento para sus años de retiro, al mismo tiempo que menos del $30 \%$ de la población adulta mayor en el Ecuador poseen un ingreso fijo al finalizar el mes, el resto debe buscar la manera de conseguir ingresos los mismos que por la pandemia del COVID-19 que se atraviesa, son prácticamente imposibles de conseguir por el confinamiento y las restricciones.

Con lo antes descrito se deduce que las políticas y programas instauradas por los gobiernos para lograr la accesibilidad a los servicios de salud son poco eficaces y más aún en tiempos de COVID-19 que el Estado 
ecuatoriano establece un plan atención domiciliaria a personas adultas mayores. Es importante señalar que en el Ecuador la población mayor a 65 años de edad asciende a 1.049.824 de personas que corresponde al 6,5\% del total de la población. En el año 2020 se espera que incremente al 7,4\% y para el año 2054 se prevé que representen el $18 \%$ de la población; para las mujeres la esperanza de vida será mayor con 83,5 años comparado con los 77,6 años de los hombres. En este período de vida, existe mayores limitaciones para acceder a recursos de subsistencia y se incrementa las necesidades de atención, así también se determina que el $45 \%$ (424.824) de la PAM se encuentra en condiciones de pobreza y extrema pobreza, el $42 \%$ (395.180) vive en el sector rural, el $14,6 \%$ de hogares pobres se compone de un adulto mayor viviendo solo, el 14,9\% son víctimas de negligencia y abandono, el 74,3\% no accede al seguro social. (INEC,2013).

Se puede deducir que la PAM, a medida que transcurre los años, se incrementa por el aumento en la esperanza de vida de las personas, así también, se colige que las políticas integrales implementadas por el Estado ecuatoriano tienen deficiencias debido que existe un alto porcentaje de personas adultas mayores que se encuentran en condiciones de pobreza y extrema pobreza; como se puede apreciar en los párrafos que anteceden y encuestas, estadísticas realizadas por diferentes instituciones, un factor importante para el no acceso a un servicio de salud es la situación económica.

\section{Conclusiones}

Alcanzar una vejez digna para los adultos mayores es un proceso constante, arduo y dinámico. El Estado ecuatoriano no ha podido alcanzar este objetivo para sus ciudadanos adultos mayores debido a políticas públicas poco eficaces, sobre todo en el ámbito de la salud, por lo que es necesario ampliar la cobertura y fortalecer la calidad de los servicios de atención integral a personas adultas mayores.

En gran parte, los patrones de salud y bienestar al interior del país se deben a los altos niveles de pobreza y desigualdad, tanto en los ingresos como en el acceso y uso de los servicios de salud, situación que se agudiza en el Ecuador por las defectuosas políticas orientadas al bienestar de los adultos mayores.

Es crítico el acceso a servicios de salud apropiados, adecuados y aceptados, debido a una serie de factores como las desfavorables condiciones socioeconómicas de un gran porcentaje de población adulta mayor, a la no existencia de los servicios o el estar incompletos, a las dificultades económicas por costos y a la no disponibilidad de seguros de salud.

Se concluye que existen deficiencias en el sistema de salud para lograr un efectivo acceso al derecho a la salud gratuita, prioritaria y especializada, debido que existe burocracia para acceder al servicio, el personal que atiende a este grupo de personas vulnerables no son especialistas o existen muy pocos especialistas en el área, además por parte del Estado y las Universidades no existe un incentivo hacia los jóvenes para que se especialicen en geriatría y/o gerontología.

Se concluye que para mejorar la atención y accesibilidad al servicio sanitario y para cumplir con el derecho que tiene la población adulta mayor a medicamentos y servicio de salud especializada y gratuita, se implementen campañas de atención preventiva domiciliaria por parte del Ministerio de Salud, reducir o eliminar la burocracia por parte de los Centros y Hospitales de Salud Pública y que el Ministerio de Salud y las Universidades debrían aunar esfuerzos para promover la especialización de los profesionales en geriatría y gerontología.

Cabe señalar que el MIES junto con el Ministerio de Educación pueden incentivar a los jóvenes para que desde sus inicios su meta sea el estudio de geriatría. El Ministerio de Inclusión Económica y Social al mismo tiempo con el Ministerio de Salud Pública capaciten de manera permanente a los profesionales que se encuentran al servicio de las personas adultas mayores y que el Ministerio de Economía y Finanzas promueva la eliminación de las 
desigualdades de acceso por razones económicas implementando programas para mejorar la economía PAM; y finalmente, el MIES, mediante campañas de concientización, estimular a las familias que sientan empatía por sus abuelitos y no los abandonen a su suerte. Tarde o temprano, todos seremos parte de este grupo vulnerable.

\section{Referencias bibliográficas}

Abarca A. (2002). Las políticas públicas como perspectiva de análisis. Revista de Ciencias Sociales, Universidad de Costa Rica, Número 97, 2002 (III).

Aguilar B. (2018). Gestión de políticas públicas educativas: Una caracterización en Norte de Santander (Colombia).

Asamble Nacional de la Republica del Ecuador (2019). Ley Orgánica de las Personas Adultas Mayores, Registro oficial 484.

Asamblea Nacional de la Republica del Ecuador (2008). Constitución de la República del Ecuador [CRE], Registro Oficial N.449.

Centro de Estudios de Población y Desarrollo Social (2005). Encuesta Demográfica y de Salud Materna Infantil (ENDEMAIN). Recuperado de https://cssr-ecuador.org/downloads/2016/11/32.-Encuesta-Demografica-yde-salud-materna-e-infantil-ENDEMAIN.pdf.

Cicogna, M. (2020). Estado, sociedad y políticas públicas. Eudeba. Recuperado de https://elibro.puce.elogim.com/es/ereader/puce/153611? Page=29 (Oszlak y O’Donnell, 1982: 112).

Comisión Interamericana de Derechos Humanos (1988). lus et Praxis, 25 (1), 220-222.

Congreso Nacional del Ecuador, Ley del Anciano (2006), Registro Oficial 376, recuperado, https://www.igualdad.gob.ec/wp-content/uploads/downloads/2017/11/ley_anciano.pdf

Convención Interamericana de Derechos Humanos (1977). Recuperado en https://biblioteca.defensoria.gob.ec/bitstream/37000/456/1/Convenci\%C3\%B3n\%20Interamericana\%20s obre\%20Derechos\%20Humanos.pdf , 19 de abril de 1978. lus et Praxis, 12(1), 275-296.

Dabove, M. I. (2013). Ciudadanía y derechos fundamentales de las personas mayores: de las políticas gerontológicas al Derecho de la Vejez. Revista de la Facultad de Derecho, 4(1).

Declaración Universal de Derechos Humanos (1948), https://www.un.org/es/documents/udhr/UDHR_booklet_SP_web.pdf

Declaración Universal de los Derechos del Hombre, (1948). lus et Praxis, 22 (1), 214-215.

Duval G. (2019), Cumplimiento de metas de políticas públicas y el acceso al servicio de salud en el Hospital de Especialidades Básicas, Universidad Cesar Vallejo.

Freire, W. (2010). Encuesta Nacional de Salud, Bienestar y Envejecimiento SABE I, Ecuador 2009-2010. Quito: Aliméntate Ecuador, Universidad San Francisco de Quito, recuperado https://www.gerontologia.org/portal/archivosUpload/Ecuador-Encuesta-SABE-presentacionresultados.pdf

García Pedroza F.; de la Fuente Rocha J; Irigoyen Coria A.; Peñaloza López Y.R., (2013) Propuesta para actualizar las políticas de atención integral a la salud en personas mayores de 65 años de edad en México, Atención Familiar, Volumen 20, 69-70, ISSN 1405-8871, https://doi.org/10.1016/S1405-8871(16)30093-1.

Guzmán J. (2006). Envejecimiento y Desarrollo en América Latina y el Caribe. CEPAL/CELADE. Serie Población y Desarrollo $N^{\circ} 28$. Santiago de Chile. 
Hernández R, http://observatorio.epacartagena.gov.co/wp-content/uploads/2017/08/metodologia-de-lainvestigacion-sexta-edicion.compressed.pdf

Huenchuan (2009). Derechos Humanos y Políticas Públicas, Comisión Económica para América Latina y el Caribe, ISBN: 9789213232156, (CEPAL) Santiago de Chile.

INEC, Instituto Nacional de Estadisticas y Censos (2011). Censo de Población y Vivienda del Ecuador.

Ministerio de Inclusión Económica y Social (2014). Reflexiones sobre la Intergeneracional dad en Ecuador. ISBN: 9789942075710.

Ministerio de Inclusión Económica y social, Agenda de igualdad para 2012 - 2013 Adultos Mayores, Quito Ecuador. Recuperado: https://fiapam.org/wp-content/uploads/2013/06/Agendas_ADULTOS.pdf

Ministerio de Salud Pública (MSP) Política Nacional de Medicamentos. Recuperado https://www.salud.gob.ec/wp-content/uploads/2017/03/Politica-Nacional-de-Medicamentos-conacuerdo.pdf, consultado el 01/03/2021.

Montenegro G, et al.(2020). Modelos para el análisis de políticas públicas en salud reportados en publicaciones científicas. Gac Sanit. 2020. https://doi.org/10.1016/j.gaceta.2019.11.007

Organización Panamericana de Salud (1997): "La Cooperación de la OPS ante los procesos de Reforma del Sector Salud", Washington DC, (esp. e eng.).

Organización Iberoamericana de Seguridad Social (2007). Situación y demandas de las personas mayores en los países del Cono Sur, Apuntes para un diagnóstico, Proyecto sobre personas mayores, dependencia y servicios sociales en los países del Cono sur, Madrid.

Organización Internacional del Trabajo (2002). Resolución y conclusiones relativas a la seguridad social, Seguridad social: un nuevo consenso, Conferencia Internacional del Trabajo, 89a. reunión, Ginebra.

Pacto de Derechos Económicos, Sociales y Culturales (1966), https://www.ohchr.org/sp/professionalinterest/pages/cescr.aspx

Parodi J. \& Runzer F. (2020). Amor en los Tiempos del COVID-19:Centrado en el cuidado de las personas mayores. Recuperado https://cmhnaaa.org.pe/ojs/index.php/rcmhnaaa/article/view/628/318, DOI: https://doi.org/10.35434/rcmhnaaa.2020.131.628

Programa de las Naciones Unidas para el Desarrollo, Informe sobre Desarrollo Humano (2011) recuperado file://C:/Users/Hp/Downloads/HDR_2011_ES_Complete.pdf

Protocolo de San Salvador (1988), https://www.oas.org/es/sadye/inclusion-social/protocolossv/docs/protocolo-san-salvador-es.pdf

Registros Administrativos diciembre (2013). IESS, ISSFA, ISSPOL, VAMS, diciembre 2013, proyección 2013, INEC. Dirección Población Adulta Mayor - Ministerio de Inclusión Económica y Social (inclusion.gob.ec)

Zafra E., Pairó R., Ramón N., Álvarez C., Borrell C., (2006), Análisis de la formulación de las políticas sobre envejecimiento en los planes autonómicos sociosanitarios y de atención a las personas mayores en España, Gaceta Sanitaria, Volumen 20, 295-302, ISSN 0213-9111, https://doi.org/10.1157/13091145.

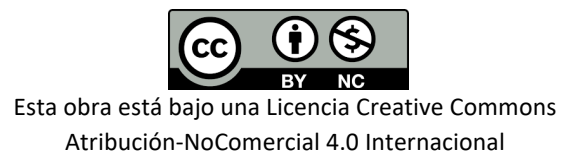

